\title{
Intermediate adhesion maximizes fluidity and migration velocity of multicellular clusters
}

\author{
${ }_{5}$ U. Roy, A. Mugler
}

${ }_{6}$ ABSTRACT Collections of cells exhibit coherent migration during morphogenesis, cancer metastasis, and wound healing. In 7 many cases, bigger clusters split, smaller sub-clusters collide and reassemble, and gaps continually emerge. The connections $\therefore$ between cell-level adhesion and cluster-level dynamics, as well as the resulting consequences for cluster properties such as 9 migration velocity, remain poorly understood. Here we investigate collective migration of one- and two-dimensional cell clusters

${ }_{10}$ that collectively track chemical gradients using a mechanism based on contact inhibition of locomotion. We develop both a 11 minimal description based on the lattice gas model of statistical physics, and a more realistic framework based on the cellular ${ }_{12}$ Potts model which captures cell shape changes and cluster rearrangement. In both cases, we find that cells have an optimal ${ }_{13}$ adhesion strength that maximizes cluster migration speed. The optimum negotiates a tradeoff between maintaining cell-cell ${ }_{14}$ contact and maintaining cluster fluidity, and we identify maximal variability in the cluster aspect ratio as a revealing signature.

${ }_{15}$ Our results suggest a collective benefit for intermediate cell-cell adhesion.

SIGNIFICANCE Cells have been observed to migrate faster and more efficiently in clusters than as individuals. We conjecture that adhesion among cells and with the extracellular environment plays an important role in achieving higher speed for the entire cluster. We carry out our analyses analytically and computationally, by employing a simplistic onedimensional model and a realistic two-dimensional model which capture the essential features of multicellular migration. Our study demonstrates that an optimal cell-cell adhesion, which corresponds to maximal cellular rearrangement and loose packing, leads to a higher migration velocity for a multicellular cluster, acting as a crucial factor in effective movement of a collection of cells in a coordinated and directed fashion.

\section{${ }_{23}$ INTRODUCTION}

${ }_{24}$ Collective cell migration is of critical importance in nearly ${ }_{25}$ all stages of life (1). Biological processes like embryoge${ }_{26}$ nesis, morphogenesis, neurogenesis, regeneration, wound ${ }_{27}$ healing, and disease propagation such as cancer metastasis 28 involve numerous cells acting in a coordinated way (1-3). ${ }_{29}$ Studies have demonstrated that multicellular clusters can 30 sense chemoattractants more efficiently and precisely than ${ }_{31}$ their isolated constituent cells do $(4,5)$. Sensory information ${ }_{32}$ is combined with mechanochemical mechanisms, including ${ }_{33}$ actin polymerization and contact-dependent polarity (known ${ }_{34}$ as contact inhibition of locomotion, CIL) $(4,6)$, to pro${ }_{35}$ duce directional migration. Recent studies have indicated that ${ }_{36}$ cadherin- and integrin-based adhesions at cell-cell junctions 37 and cell-extracellular matrix (ECM) contacts respectively are ${ }_{38}$ indispensable for migration of multicellular clusters $(1,7,8)$ ${ }_{39}$ Cell-cell and cell-ECM adhesion are integrated with actin 40 dynamics to keep clusters together during collective cell 41 migration $(1,9)$.

42 Collective migration presents a mechanical tradeoff, as ${ }_{43}$ cells must negotiate a balance between displacing themselves ${ }_{44}$ with respect to the ECM, but not separating themselves from ${ }_{45}$ other cells. In many cases this results in clusters that are
${ }_{46}$ dynamic and loosely packed rather than rigidly structured. For ${ }_{47}$ example, in the case of neural crest cells, a group of pluripo48 tent cells in all vertebrate embryos that can migrate very long ${ }_{49}$ distances, bigger clusters split, smaller sub-clusters collide ${ }_{50}$ and reassemble, and gaps continually appear and disappear ${ }_{51}(4,10)$. This raises the question of whether there is an interme52 diate, rather than very strong or weak, adhesion strength that ${ }_{53}$ optimally negotiates this tradeoff and results in dynamic loose 54 clustering and maximally efficient collective migration. Cell ${ }_{55}$ adhesion is clearly crucial to collective migration, but the ${ }_{56}$ mechanisms are not yet well understood.

57 Here we use mathematical modeling and simulation to in58 vestigate the role of cell-cell and cell-ECM adhesion strength 59 in determining collective migration efficiency and the con60 comitant effects on cluster shape and dynamics. Rather than ${ }_{61}$ focusing on the details of the mode of action or molecular 62 properties of different types of adhesion molecules, we de${ }_{63}$ velop a generic model which explores the different regimes ${ }_{64}$ of adhesion strength, so that we may have a general under65 standing of the phenomena. We start with a one-dimensional ${ }_{66}$ model based on the lattice gas model of statistical physics (11) ${ }_{67}$ that allows us to analytically probe the collective migration 68 velocity of a linear chain of cells as a function of adhesion 
U. Roy and A. Mugler

69 strength. We then extend this model to two dimensions using 70 the cellular Potts model (12-14), which more realistically 71 captures cell shape, cluster rearrangement, and other essential 72 aspects of cluster migration.

Numerical results from both the one- and the two-dimensional 74 model suggest the existence of an intermediate adhesion 75 strength among cells that leads to the fastest migration of 76 a multicellular cluster. Specifically, there exists a regime of 77 intercellular and cell-ECM adhesion strengths which corre78 sponds to optimally effective migration. We demonstrate that, 79 in this regime, the clusters possess the maximal rearrangeso ment capacity while remaining as a connected cluster, rather ${ }_{81}$ than falling apart and scattering into single isolated cells or 82 strongly sticking together as a compact structure.

\section{${ }_{83}$ METHODS}

${ }_{84}$ We first consider a simplified one-dimensional model for ${ }_{85}$ collective migration based on the lattice gas model of statistical ${ }_{86}$ physics, and then a more realistic two-dimensional model ${ }_{87}$ based on the cellular Potts model. Here we first review the 88 lattice gas model (later, in the Results section, we discuss 89 our new calculations using this model, as well as our own ${ }_{90}$ modifications to it). We then present the model details of the ${ }_{91}$ cellular Potts model.

\section{${ }_{92}$ One-dimensional lattice gas model}

${ }_{93}$ We first investigate a one-dimensional collective of cells using ${ }_{94}$ the lattice gas model. Consider $N$ cells arranged in a one${ }_{95}$ dimensional lattice of $V$ sites with $V \geq N$ (Fig. 1A). $\sigma_{i}$ ${ }_{96}$ denotes the state of each lattice site $i . \sigma_{i}=1$ represents a cell ${ }_{97}$ while ECM is labeled by $\sigma_{i}=0$.

$98 \quad$ Assume that interaction exists only between adjacent cells; 99 the total energy for a given configuration of cells $\left\{\sigma_{i}\right\}$ can 100 then be expressed as

$$
E_{L G}=-\epsilon \sum_{i=1}^{V} \sigma_{i} \sigma_{i+1}
$$

${ }_{101}$ where $-\epsilon$ is the interaction energy between two adjacent ${ }_{102}$ cells representing their adhesion. We impose $\sigma_{V+1}=\sigma_{1}$ for ${ }_{103}$ periodicity and $\sum_{i=1}^{V} \sigma_{i}=N$ to conserve cell number.

104 The grand partition function for the lattice gas is

$$
\Xi_{L G}=\sum_{N=0}^{V} z^{N} Z_{L G}
$$

${ }_{105}$ where $Z_{L G}=\sum_{\left\{\sigma_{i}\right\}} e^{-\beta E_{L G}}$ is the canonical partition func106 tion, $z=e^{\beta \mu}$ is the fugacity parameter, with $\beta=\left(k_{B} T\right)^{-1}$ and ${ }_{107} \mu$ denoting the chemical potential. The inverse of Eq. (2) is

$$
Z_{L G}=\frac{1}{N !} \frac{\partial^{N}}{\partial z^{N}} \Xi_{L G}
$$
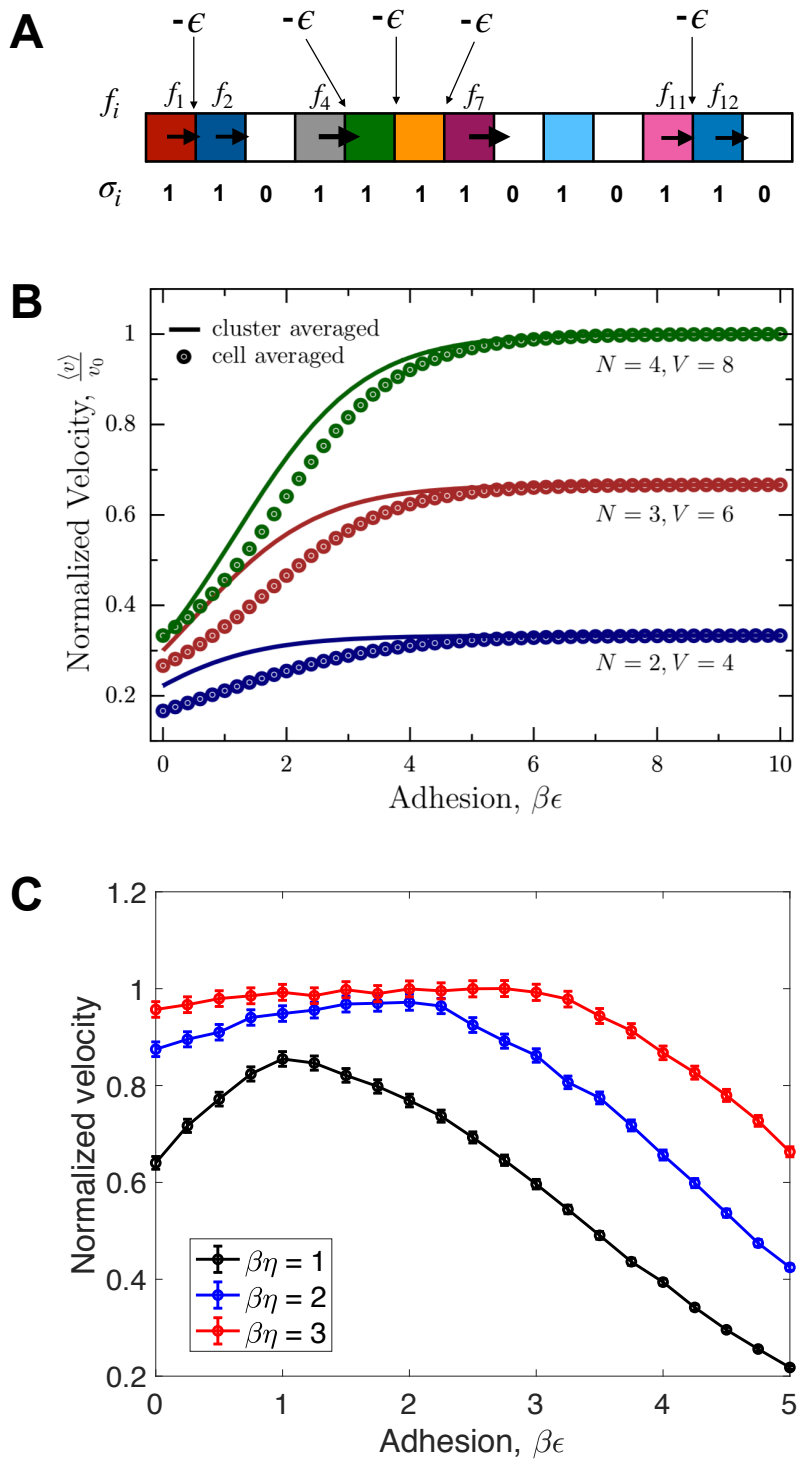

Figure 1: Velocity vs. adhesion for one-dimensional collective cell migration. A. Schematic showing a collection of cells (colors, $\sigma_{i}=1$ ) and ECM (white, $\sigma_{i}=0$ ) arranged in a linear chain. Each pair of cells has an interaction energy $-\epsilon$. Arrows indicate motility force $f_{i}$. B. Normalized velocity $\langle v\rangle / v_{0}$ as a function of adhesion $\beta \epsilon$ for the undriven model, Eq. (1). C. Normalized velocity as a function of adhesion $\beta \epsilon$ for the driven model, Eq. (16). 
${ }_{108}$ Inserting Eq. (1) into Eq. (2) and exploiting the fact that ${ }_{109} N=\sum_{i=1}^{V} \sigma_{i}$, Eq. (2) can be recast as

$$
\Xi_{L G}=\sum_{\left\{\sigma_{i}\right\}} \exp \left(\beta \epsilon \sum_{i=1}^{V} \sigma_{i} \sigma_{i+1}+\beta \mu \sum_{i=1}^{V} \sigma_{i}\right)
$$

${ }_{110}$ We now recognize that the grand partition function of the 111 lattice gas model as expressed in Eq. (4) has the same form as 112 the canonical partition function of the Ising model $(11,15)$. ${ }_{113}$ Specifically, relating the $\sigma_{i} \in\{0,1\}$ to Ising spin variables ${ }_{114} s_{i} \in\{-1,1\}$ via $\sigma_{i}=\left(s_{i}+1\right) / 2$, Eq. (4) reads

$$
\Xi_{L G}=Z_{I} e^{\beta \mu V / 2} e^{\beta \epsilon V / 4}
$$

${ }_{115}$ where $Z_{I}$ is the canonical partition function of the Ising 116 model with magnetic field $H=(\epsilon+\mu) / 2$ and coupling energy ${ }_{117} J=\epsilon / 4$.

${ }_{118}$ The canonical partition function of the Ising model is ${ }_{119}$ exactly solvable in one dimension and reads

$$
Z_{I}=\lambda_{+}^{V}+\lambda_{-}^{V}
$$

120 for a periodic chain, where

$$
\lambda_{ \pm}=e^{\beta J} \cosh (\beta H) \pm \sqrt{e^{2 \beta J} \sinh ^{2}(\beta H)+e^{-2 \beta J}} .
$$

${ }_{121}$ Thus, Eqs. (3) and (5)-(7) constitute an analytic expression 122 for the canonical partition function of the lattice gas model. ${ }_{123}$ We use this fact to calculate the cluster migration velocity in ${ }_{124}$ the Results section.

\section{${ }_{225}$ Two-dimensional cellular Potts model}

${ }_{126}$ To more realistically model cluster migration in two dimen${ }_{127}$ sions, we use computer simulation. Many cellular automata 128 models have been developed for this task (16-18); we use ${ }_{129}$ the cellular Potts model (CPM) $(19,20)$. The CPM captures 130 realistic properties such as changes in cell shape and cell 131 size, rearrangement of cells within a cluster, and the dynamic 132 breakup or re-aggregation of sub-clusters. Diverse biological ${ }_{133}$ phenomena like chemotaxis, cell sorting, endothelial cell ${ }_{134}$ streaming, tumor invasion and cell segregation have been 135 modeled using the CPM $(19,21,22)$.

${ }_{136}$ We have considered a discrete two-dimensional lattice. ${ }_{137}$ Each cell is represented by a group of lattice sites $x$ with the ${ }_{138}$ same integral values for their lattice labels $\sigma(x)>0$ (Fig. 2). ${ }_{139}$ The empty lattice sites correspond to the extra-cellular matrix ${ }_{140}(\mathrm{ECM})$, with lattice label $\sigma(x)=0$, providing an environment 141 through which the cells move. The initial configuration has 142 several cells arranged in a single cluster. The energy of the ${ }_{143}$ whole system $E_{C P M}$ has contributions from two factors: the ${ }_{144}$ first one is the adhesion while the second one is the area 145 restriction term,

$$
E_{C P M}=\sum_{\left\langle x, x^{\prime}\right\rangle} J_{\sigma(x), \sigma\left(x^{\prime}\right)}+\sum_{i=1}^{N} \lambda_{A}\left(\delta A_{i}\right)^{2}
$$

${ }_{146}$ The adhesion energy term $J_{\sigma(x), \sigma\left(x^{\prime}\right)}$ is given by the following 147

$$
J_{\sigma(x), \sigma\left(x^{\prime}\right)}=\left\{\begin{array}{lll}
0 & \sigma(x) \sigma(x) \geq 0 & \text { within ECM or same cell, } \\
\alpha & \sigma(x) \sigma\left(x^{\prime}\right)=0 & \text { cell-ECM contact } \\
\gamma & \sigma(x) \sigma\left(x^{\prime}\right)>0 & \text { cell-cell contact. }
\end{array}\right.
$$

${ }_{148} \alpha$ denotes the interaction strength of any cell due to adhe${ }_{149}$ sion with its environment while intercellular adhesiveness is ${ }_{150}$ characterized by $\gamma$. A migrating cell is refrained from grow151 ing or shrinking to unphysical sizes, as well as branching or 152 stretching into unphysical shapes, due to the presence of the ${ }_{153}$ area restriction term in Eq. (8). Cells undergo fluctuations ${ }_{154}$ in size $\delta A_{i}$ around a desired area $A_{0}$ via $\delta A_{i} \equiv A_{i}(t)-A_{0}$. ${ }_{155}$ We have set $\lambda_{A}$ to be unity (23). Previous work (12-14, 23) 156 has included a perimeter restriction term in addition to the 157 area restriction term. For simplicity we omit this term, as ${ }_{158}$ we find that sufficiently large $\alpha$ and $\gamma$ constrain perimeter by 159 cell-ECM or cell-cell contact.

${ }_{160}$ Our model of migration is based on contact inhibition of ${ }_{161}$ locomotion (CIL), a well known and central mechanism of 162 collective cell movement (6). The formation of cell protrusions ${ }_{163}$ is locally inhibited when a cell comes into contact with another ${ }_{164}$ cell, and hence the cell ceases to move in that direction. Instead, 165 the cell generates protrusions away from the site of contact ${ }_{166}(24,25)$, which produces force in the outward direction. Direct ${ }_{167}$ evidence of CIL has been observed in migrating clusters, 168 where outer cells have strong outward polarization while inner ${ }_{169}$ cells weakly protrude (4). Note that under this mechanism, 170 directional migration is purely collective: two or more cells 171 in contact are polarized, whereas single isolated cells are not. ${ }_{172}$ We consider the case where cells exist in an external ${ }_{73}$ chemical gradient. Drosophila egg chamber cells (26-29), 74 clusters of lymphocytes (30), neural crest cells (4), and ep75 ithelial organoids (5) exhibit emergent gradient sensing and ${ }_{76}$ collective migration in response to graded chemical cues. 177 Under the assumption that the chemical concentration influ178 ences the magnitude of the protrusive forces, the presence 179 of a chemical gradient creates a force imbalance $(31,32)$, 180 allowing the cluster to respond to the gradient. However, as a 181 cluster migrates up a gradient according to this mechanism, 182 the background concentration increases, which increases the 183 outward forces and can cause the cluster to scatter (31). To pre184 vent scattering, we adopt an adaptive mechanism of gradient 185 sensing $(5,23,31)$, in which cells respond to the difference 186 between the local chemical concentration and the average 187 experienced over the entire cluster. Evidence for adaptive ${ }_{188}$ collective gradient sensing has been observed in epithelial 189 organoids (5).

Specifically, we take the magnitude of the force experi191 enced by cell $i$ to be

$$
F_{i}=\eta g\left(x_{c m}^{i}-x_{c c m}\right)
$$

192 where $\eta$ sets the force strength, $g$ is the concentration gradient 193 which is in the $x$ direction (downward in Fig. 2 and subsequent 

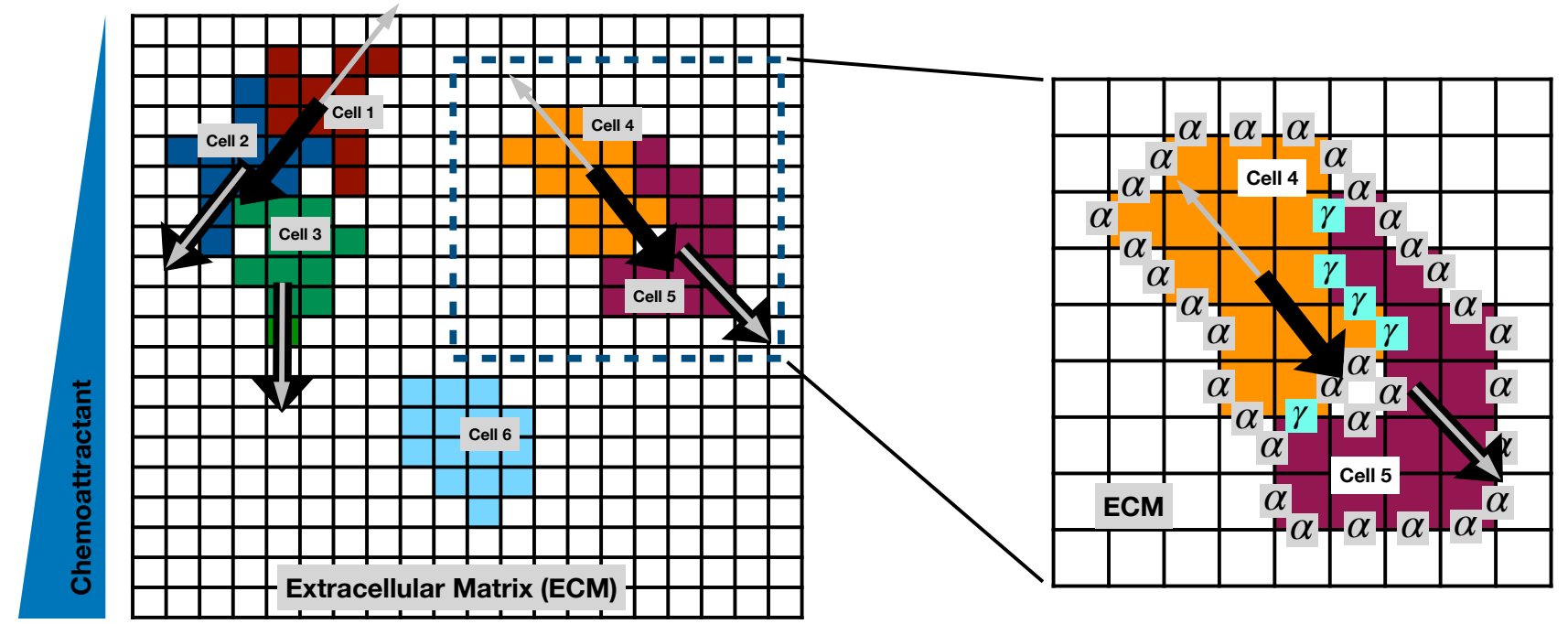

Figure 2: Cellular Potts model for collective migration in a chemical gradient. A schematic of the adaptive cellular Potts model (CPM) depicting a characteristic snapshot of three multicellular clusters of different sizes. The cluster consisting of two cells, enclosed within a dashed box (left), is zoomed (right) to show cell-cell energy penalty $\gamma$ and cell-ECM energy penalty $\alpha$. All cells have respective motility force vectors (black arrows) and repulsion vectors (gray arrows; away from cell-cell contact as a result of CIL) in a linear chemoattractant gradient. A single isolated cell (cell 6) has no force acting on it since we have considered CIL as our guiding mechanism for motility.

194 figures), $x_{c m}^{i}$ and $x_{c c m}$ are the $x$ coordinates of the center-of- ${ }_{218}$ with probability $P$, given by

195 mass of the cell and of the whole cluster respectively, and 196 the subtraction expresses the adaptivity. We set $\eta g=1$ in 197 this work. The direction of the force experienced by cell ${ }_{198} i$ is determined according to CIL (23): we sum all vectors 199 pointing from cell-pixels in contact with any other cell to the ${ }_{200}$ center-of-mass of cell $i$. This net 'repulsion' vector points ${ }_{201}$ outward (gray in Fig. 2), whereas the force direction is flipped 202 when the sign of Eq. (10) is negative (black in Fig. 2). The ${ }_{203}$ forces contribute a work term to the energy functional, given 204 by

$$
W=-\sum_{i=1}^{N} \vec{F}_{i} \cdot \Delta \vec{x}_{i}
$$

${ }_{205}$ where $\Delta \vec{x}_{i}$ is the change in the center-of-mass of each cell ${ }_{206}$ upon a configurational change, discussed next.

$$
P= \begin{cases}e^{-\left(\Delta E_{C P M}+W\right)} & \Delta E_{C P M}+W \geq 0 \\ 1 & \Delta E_{C P M}+W<0,\end{cases}
$$

219 where $\Delta E_{C P M}$ is the change in energy of the system due to ${ }_{220}$ the attempted move, calculated from Eq. (8), and $W$ is the 221 bias term given by Eq. (11).

\section{${ }_{222}$ RESULTS}

\section{${ }_{223}$ Driven lattice gas model exhibits optimal cell-cell adhesion}

${ }_{225}$ We first consider the one-dimensional lattice gas model (Meth226 ods) and ask how the average cell velocity depends on the ${ }_{227}$ adhesion strength. As in the CPM described above, we assume ${ }_{228}$ that the force $\left(f_{i}\right.$ in Fig. 1A) is exerted by the edge cells due ${ }_{207}$ Given the energy and work terms, cellular dynamics under 229 to CIL and is proportional to the local concentration of an ${ }_{208}$ the CPM are simulated using a Monte Carlo process which ${ }_{230}$ external chemical. In one dimension, there are only two edge ${ }_{209}$ is based on the principle of minimizing the energy of the ${ }_{231}$ cells per sub-cluster of at least two cells (single isolated cells 210 whole system. Specifically, motility is modeled by an addition 232 experience no contacts and therefore no force). In a linear 211 (copying the identity of one cell-pixel, chosen randomly, to ${ }_{233}$ chemical profile, the net force will be proportional to the linear ${ }_{212}$ its neighboring site) or removal (copying an ECM-pixel to ${ }_{234}$ extent of the sub-cluster, equivalent to the number of cell-cell ${ }_{213}$ a site previously occupied by cell) of pixels. Each Monte ${ }_{235}$ contacts. Assuming that the velocity is proportional to the ${ }_{214}$ Carlo step selects randomly a pair of adjacent lattice sites, and ${ }_{236}$ force (appropriate at low Reynolds number), the velocity of ${ }_{215}$ attempts to copy the identity of one to the other. It calculates ${ }_{237}$ a sub-cluster can be expressed as $v_{0} \sum_{i} \sigma_{i} \sigma_{i+1}$, where the ${ }_{216}$ the energy of the previous (before copying) and the new (after ${ }_{238}$ sum extends over the indices of the sub-cluster, and $v_{0}$ is an ${ }_{217}$ copying) configuration. The new configuration is accepted ${ }_{239}$ arbitrary constant that sets the velocity scale. The average 
${ }_{240}$ velocity over all sub-clusters in a particular configuration $\left\{\sigma_{i}\right\}$ ${ }_{241}$ is the sum of all such terms divided by the total number of 242 sub-clusters, or

$$
v=\frac{v_{0} \sum_{i=1}^{V} \sigma_{i} \sigma_{i+1}}{\sum_{i=1}^{V} \sigma_{i}\left(1-\sigma_{i+1}\right)}=-\frac{v_{0} E_{L G}}{\epsilon N+E_{L G}} .
$$

${ }_{243}$ Here the denominator counts sub-clusters by their rightmost ${ }_{244}$ edges, and the second step recalls Eq. (1). We have chosen to 245 weight each cluster equally in Eq. (13) for analytic tractability, 246 but we will see that similar results are obtained if each cell is ${ }_{247}$ weighted equally instead, as in later Results sections.

${ }_{248}$ The average velocity is the sum of Eq. (13) against the 249 Boltzmann probability,

$$
\langle v\rangle=\sum_{\left\{\sigma_{i}\right\}} \frac{-v_{0} E_{L G}}{\epsilon N+E_{L G}} \times \frac{e^{-\beta E_{L G}}}{Z_{L G}}=\frac{v_{0}}{Z_{L G}} \sum_{n=0}^{\infty}\left(\frac{\partial_{\beta}}{\epsilon N}\right)^{n} Z_{L G} .
$$

${ }_{250}$ The second step recognizes that $n$ derivatives of the partition ${ }_{251}$ function extract $n$ powers of $-E_{L G}$, which when summed as ${ }_{252}$ a geometric series are equivalent to the first expression. Eq. ${ }_{253}$ (14) connects the average velocity with the canonical partition ${ }_{254}$ function of the lattice gas, for which we have an analytic 255 expression (Methods).

${ }_{256}$ Eq. (14) depends on the size of the lattice $V$, the number of ${ }_{257}$ cells $N$, the velocity scale $v_{0}$, and the dimensionless adhesion ${ }_{258}$ energy $\beta \epsilon$. Therefore, we can ask for a given $V$ and $N$, how the 259 normalized velocity $\langle v\rangle / v_{0}$ depends on the adhesion strength ${ }_{260} \beta \epsilon$. As an example, for $V=8$ and $N=4$, Eq. (14) evaluates 261 to

$$
\frac{\langle v\rangle}{v_{0}}=\frac{4 e^{\beta \epsilon}+18 e^{2 \beta \epsilon}+12 e^{3 \beta \epsilon}}{1+12 e^{\beta \epsilon}+18 e^{2 \beta \epsilon}+4 e^{3 \beta \epsilon}} .
$$

${ }_{262}$ We see in Fig. $1 \mathrm{~B}$ (green curve) that $\langle v\rangle / v_{0}$ is a monotonically ${ }_{263}$ increasing function of $\beta \epsilon$.

${ }_{264}$ In general we find analytically that velocity increases 265 monotonically with adhesion strength for other values of ${ }_{266} N$ and $V$, and also numerically when cells are weighted ${ }_{267}$ equally in the average (Fig. 1B). This would imply that the ${ }_{268}$ optimal adhesion is infinitely strong. However, thus far, this ${ }_{269}$ model neglects the impact of the motility process itself on the 270 probability of occurrence of each configuration $\left\{\sigma_{i}\right\}$. That ${ }_{271}$ is, the probability is determined entirely by the Boltzmann ${ }_{272}$ distribution, which depends only on the adhesion energy. ${ }_{273}$ Instead, we expect that the motility forces will influence the ${ }_{274}$ ensemble of configurations, as some configurations that are 275 driven by collective movement will occur more frequently 276 than they would in the undriven system.

${ }_{277}$ To account for the influence of motility on the configura278 tion ensemble, we add a driving term to the energy function 279 that is proportional to the motility forces. Specifically, we ${ }_{280}$ consider the change in energy to be of the following form,

$$
\Delta E=\Delta E_{L G}-\eta f_{i} \Delta x .
$$

${ }_{281}$ Here $\Delta E$ is the change in energy when cell $i$ shifts to a neigh${ }_{282}$ boring lattice position. $\Delta E_{L G}$ is the change in the adhesion ${ }_{335}$
${ }_{283}$ energy according to Eq. (1), while $-\eta f_{i} \Delta x$ is the work that ${ }_{284}$ occurs when the change in cell position $\Delta x$ aligns with the 285 motility force $f_{i}$. The latter term is analogous to the work ${ }_{286}$ term in the CPM, Eq. (11). The sign of this term reflects the ${ }_{287}$ fact that the motility forces on both ends of the cluster point ${ }_{288}$ in the gradient direction, due to the adaptivity (see Methods 289 for details). We continue to take $f_{i}=n-1$ to be the number 290 of connected edges in the sub-cluster of size $n$, and $\eta$ sets the 291 strength of the motility. Note that $\eta=0$ corresponds to the 292 undriven ensemble as before.

We evolve the system via Monte Carlo simulation as in the ${ }_{29}$ CPM (Methods). We randomly choose a pair of non-identical 295 neighboring sites, i.e., a cell and an ECM site, and swap them, ${ }_{296}$ calculate the energy change following Eq. (16), and accept 297 the new configuration with Boltzmann probability $e^{-\beta \Delta E}$. ${ }_{298}$ The center-of-mass velocity averaged over many instances is 299 shown in Fig. 1C for different values of $\beta \eta$. We observe in all 300 cases that there is a clear optimum in the adhesion strength for 301 which the cluster has the maximum migration velocity. We 302 conclude that the effect of motility is to bias the ensemble of ${ }_{303}$ configurations away from its equilibrium distribution, which ${ }_{304}$ is necessary to observe an optimal adhesion strength.

The optimal adhesion strength arises due to the following ${ }_{06}$ tradeoff. On the one hand, weak adhesion results in isolated cells that diffuse without bias, except when they happen to colos lide and briefly attain a bias due to the CIL. On the other hand, 309 strong adhesion causes the first term in Eq. (16) to dominate 310 over the second, suppressing movement of cells at the leading 311 edges of sub-clusters, and therefore suppressing movement as ${ }_{312}$ a whole. The optimal adhesion strength negotiates the balance 313 between the two, resulting in clusters that are tight enough to 314 cohere but fluid enough to allow forward progress.

The one-dimensional model considered thus far captures 316 the core physics of an optimal adhesion strength but necessarily 37 neglects changes in cell and cluster shape, as well as intra18 cluster cell rearrangements, that are typical of multicellular ${ }_{319}$ migration in larger dimensions. Therefore, we use the two${ }_{320}$ dimensional CPM to investigate these aspects next.

\section{Cellular Potts model exhibits optimal cell-cell and cell-ECM adhesion}

${ }_{323}$ To capture more realistic motion of cells in two dimensions, ${ }_{324}$ we use the CPM (Methods). We plot the migration velocity ${ }_{325}$ for a cluster of nine cells in the phase space of $\alpha$, which ${ }_{326}$ represents the energy penalty for cell-ECM contact, and ${ }^{327} \gamma$, which represents the energy penalty for cell-cell contact ${ }_{328}$ (see Fig. 3A). We see a clear optimum in regime ii (red), ${ }_{329}$ corresponding to intermediate $\alpha$ and $\gamma$. We have checked 330 that the existence and location of the optimum is not strongly ${ }_{331}$ dependent on the number of cells in the system. Thus, not 332 only is there an optimal cell-cell adhesion strength $(\gamma)$ as ззз found in the one-dimensional model, there is also an optimal 34 cell-ECM adhesion strength $(\alpha)$.

The reason for the optimum is illustrated in Fig. 3B. At 


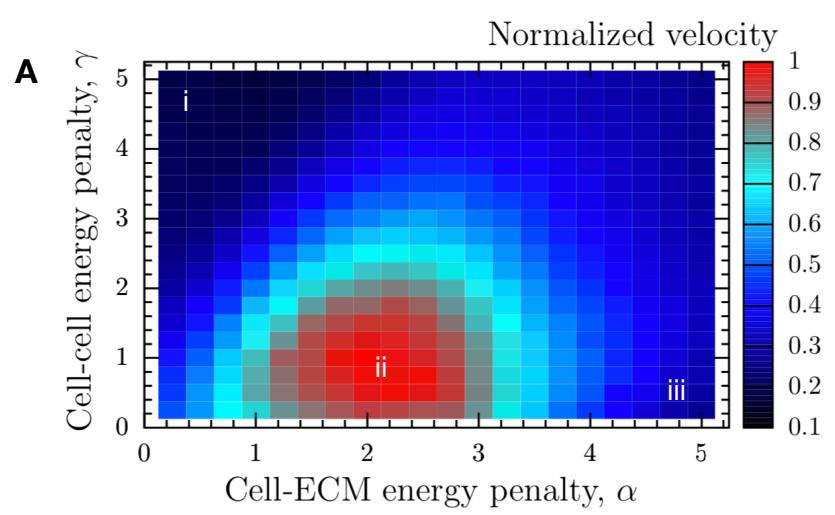

B

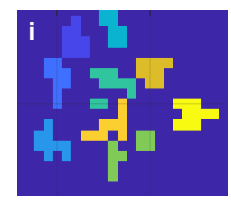

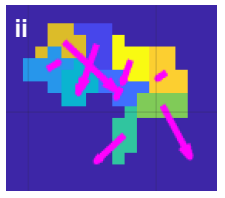

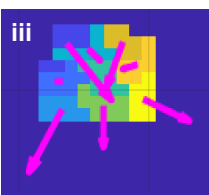

${ }_{336}$ low $\alpha$ and high $\gamma$ (region i), cells adhere to the ECM but not ${ }_{337}$ each other. Therefore, they scatter and do not benefit from the ${ }_{338}$ collective determination of the gradient direction, resulting in ззя a low velocity. At high $\alpha$ and low $\gamma$ (region iii), cells adhere 340 to each other but prefer to avoid contact with the ECM. The ${ }_{341}$ latter prevents protrusions from forming, also resulting in a 342 low velocity. Region ii optimally negotiates this tradeoff.

343 Although Fig. 3 demonstrates the existence of optimal 344 adhesion strengths, it does not directly address the question 345 of what properties of the clusters correspond to this optimum. ${ }_{346}$ As these properties could lead to experimental predictions ${ }_{347}$ and further reveal the physical mechanisms behind optimal 348 collective migration, we explore this question next.

\section{${ }_{349}$ Optimum arises from intact but maximally fluid ${ }_{350}$ clusters}

${ }_{351}$ We first hypothesized that the optimal migration velocity 352 corresponds to the transition between a fully connected cluster ${ }_{353}$ and multiple disconnected sub-clusters (Fig. 4A). Such a ${ }_{354}$ transition occurs when $\gamma \approx 2 \alpha$. The reason is that two cell 355 edges that are in contact with each other will have an energy ${ }_{356}$ cost of $\gamma$, whereas if these two edges are exposed to the ECM ${ }_{357}$ they will have an energy cost of $2 \alpha$. Thus $\gamma<2 \alpha$ will promote 358 cell scattering, while $\gamma>2 \alpha$ will promote cluster cohesion.

359 Fig. 4B confirms the transition: we see in Fig. 4B that to ${ }_{360}$ the left of the line $\gamma=2 \alpha$ (dashed) the mean sub-cluster size ${ }_{361}$ is less than the total cell number of 9 cells, whereas to the 362 right of the line it converges to 9 cells. Indeed, in the inset ${ }_{363}$ of Fig. 4B we see that far to the left of the transition (region Figure 3: Velocity vs. intercellular and cell-ECM adhesion 364 i), the sub-cluster size distribution is broad, with significant strengths for two-dimensional collective cell migration. A. ${ }^{365}$ probability to observe sub-clusters of size less than nine, Normalized center-of-mass velocity vs. cell-ECM energy 366 including isolated cells of size one. In contrast, far to the right penalty $\alpha$ and cell-cell energy penalty $\gamma$. Velocity is maximal 367 of the transition (region vi), we see that the sub-cluster size in region ii. Velocity is computed after 20,000 Monte Carlo 368 distribution has support only at nine, meaning all cells remain steps and averaged over 200 trials for each value of $\alpha$ and $\gamma$. B. ${ }^{369}$ intact throughout the migration.

Snapshots from simulation of a cluster of nine cells, illustrating $370 \quad$ The optimal velocity occurs in region ii of Fig. 3A which the cluster configuration while migrating, corresponding to 371 corresponds to region vi of Fig. $4 \mathrm{~B}$ (dashed circle), which is far different regimes in the parameter space: (i) cells scatter and 372 from the connectedness transition. Evidently, being relatively diffuse away, (ii) cells remain connected with intermediate $73^{3}$ deep within the fully connected regime is optimal for maximal adhesion, and (iii) cells tightly adhere to one another forming ${ }_{374}$ cluster velocity. Therefore, being at the transition between a compact structure. 375 connected and disconnected cannot explain the optimum ${ }_{376}$ observed in our model.

377 We next hypothesized that the optimal migration velocity 378 corresponds to the ability of the cluster to extend maximally 379 in the gradient direction while remaining intact (Fig. 5A). 380 Maximal extension would allow the cluster to span the largest ${ }_{381}$ distance in the gradient direction, meaning that the concentra382 tion difference between the front (or back) cell and the cluster ${ }_{383}$ center-of-mass would be largest. This would result in the 384 largest force exerted by these cells via Eq. (10). We quantify 385 extension using the cluster aspect ratio (AR): the ratio of the 386 length of the cluster parallel vs. perpendicular to the gradient ${ }_{387}$ direction. We see in Fig. 5B that the average aspect ratio 388 indeed varies as a function of the adhesion parameters $\alpha$ and 


\section{Connectedness}

A

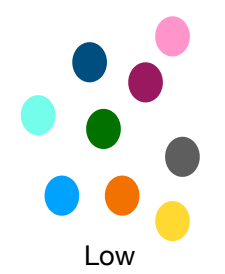

Intermediate
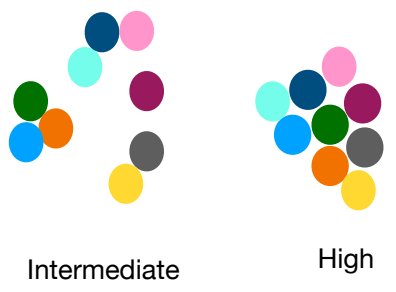

B

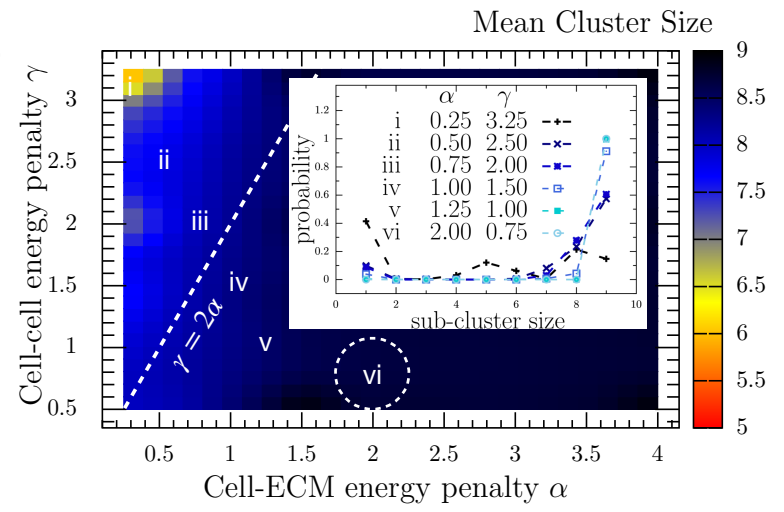

Figure 4: Connectedness transition does not account for maximal cluster velocity. A. Schematic illustrating low, intermediate, and high connectedness. B. Mean cluster size vs. $\alpha$ and $\gamma$ for 9 cells. Cells transition from disconnected to connected when $\alpha>2 \gamma$, as predicted, which is far from where velocity is maximal (dashed circle). Inset: Sub-cluster size distribution for different values of $\alpha$ and $\gamma$ (as shown by i-vi in A) clearly exhibits a transition from multiple sub-clusters to a single cluster of size nine. Sub-cluster sizes are computed over 10,000 Monte Carlo steps for each value of $\alpha$ and $\gamma$.

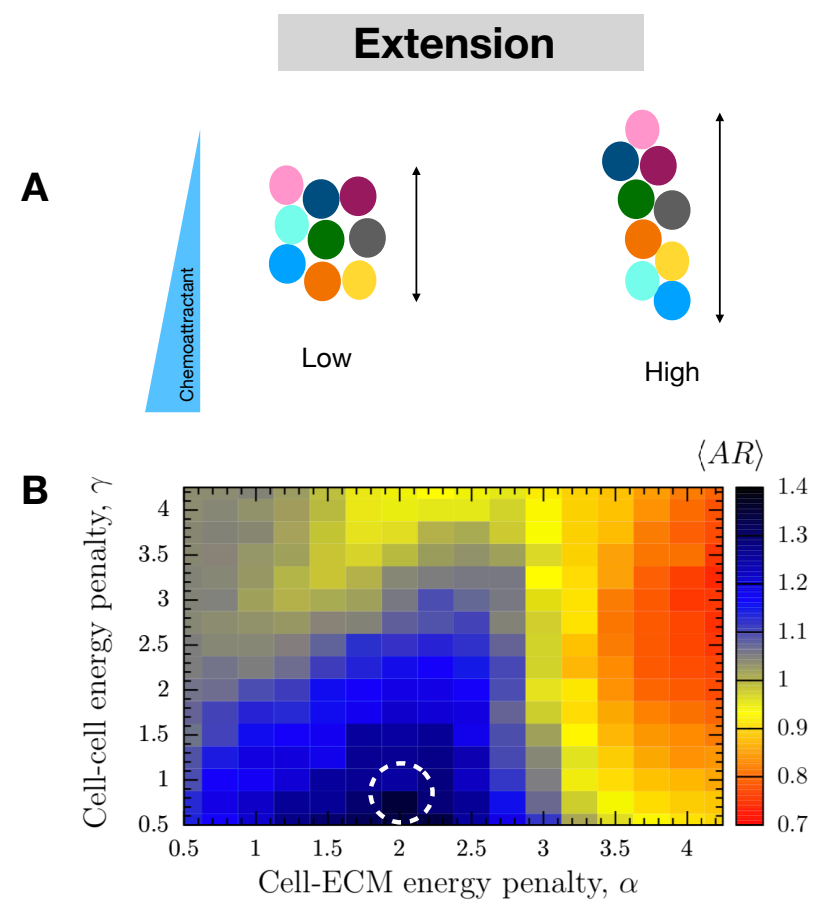

Figure 5: Extension in gradient direction correlates with cluster velocity. A. Schematic illustrating low and high cluster extension in the gradient direction, which we quantify by the aspect ratio (AR). B. Mean aspect ratio $\langle A R\rangle$ vs. $\alpha$ and $\gamma$ exhibits maximum in same location as maximal cluster velocity (dashed circle). Aspect ratio is computed over 20,000 Monte Carlo steps and averaged over 200 trials for each value of $\alpha$ and $\gamma$. 


\section{Fluidity}

A
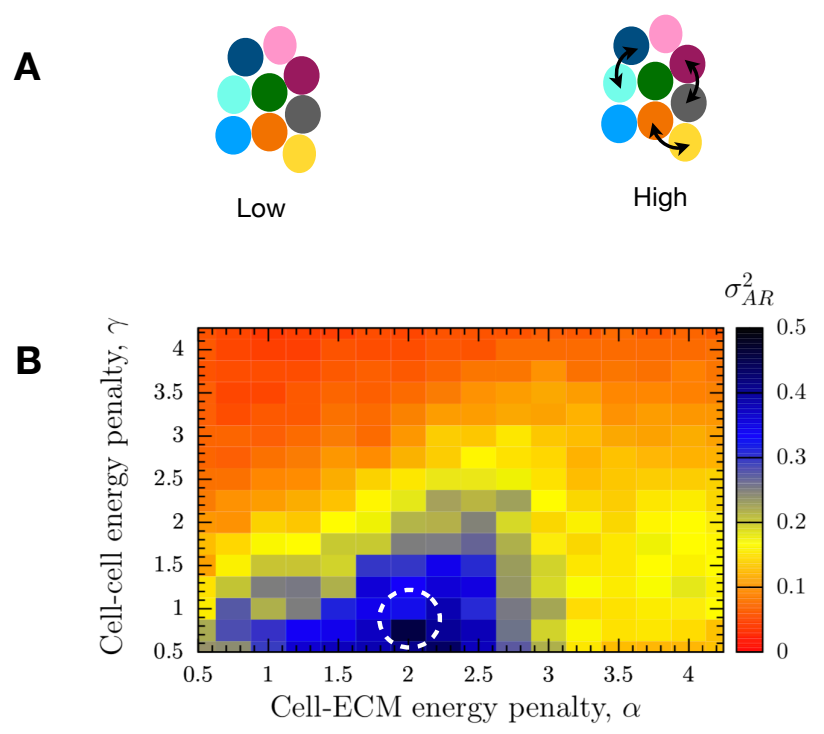

Figure 6: Cluster fluidity correlates with cluster velocity. A. Schematic illustrating low and high fluidity. High fluidity corresponds to cell rearrangement and changes in overall cluster shape, which we quantify using the variance of the aspect ratio. B. Variance of the aspect ratio vs. $\alpha$ and $\gamma$ exhibits maximum in same location as maximal cluster velocity (dashed circle). Aspect ratio is computed over 20,000 Monte Carlo steps, and variance is computed over 200 trials for each value of $\alpha$ and $\gamma$.

${ }_{389} \gamma$, and that a maximum is observed (dark blue) corresponding 390 to extension parallel to the gradient direction $(\langle A R\rangle>1)$. The 391 location of this maximum corresponds to that of the maximal 392 velocity (dashed circle in Fig. 5B). We conclude that maximal 393 cluster extension leads to maximal migration velocity.

394

The maximal average extension observed in Fig. 5B could 395 occur in multiple different ways. One possibility is that the 396 cluster relaxes to a maximally extended shape and stays 397 in this shape throughout the course of the migration. An 398 alternative possibility is that the cluster is fluid, with cells 399 free to rearrange while the cluster remains intact (Fig. 6A). ${ }_{400}$ Previous studies have shown that fluidity determines the ${ }_{401}$ properties of a jamming transition in confluent sheets (33), 402 and that more fluid multicellular clusters can be more effective ${ }_{403}$ gradient sensors (34). If the cluster is fluid, motility forces ${ }_{404}$ would then drive the cluster into a maximally extended shape ${ }_{405}$ on average, but many shapes could be visited throughout the ${ }_{406}$ migration process. We therefore expect the two possibilities ${ }_{407}$ of a rigid or a fluid cluster to have low or high variability in 408 the aspect ratio, respectively.
410 fluidity using the variance in the aspect ratio, $\sigma_{A R}^{2}$. Fig. $6 \mathrm{~B}$ ${ }_{411}$ plots $\sigma_{A R}^{2}$ as a function of $\alpha$ and $\gamma$. We see that it has a ${ }_{412}$ maximum at the same location of the optima in the migra${ }_{413}$ tion velocity and the cluster extension (dashed circle). Thus, ${ }_{414}$ maximal velocity corresponds not to a cluster that is rigidly 415 extended in the gradient direction, but to a cluster that is ${ }_{416}$ maximally fluid: extended on average, but freely exploring the ${ }_{417}$ space of cluster shapes as migration proceeds. This maximal ${ }_{418}$ fluidity is enabled at intermediate adhesion strengths: suffi${ }_{419}$ ciently strong to keep cells intact as a fully connected cluster, ${ }_{420}$ but sufficiently weak to allow maximal variability in cluster ${ }_{421}$ shape.

\section{${ }_{422}$ DISCUSSION}

${ }_{423}$ We have developed a model to investigate the role of cell${ }_{424}$ cell and cell-ECM adhesion in determining the migration ${ }_{425}$ velocity of multicellular clusters. In our model, migration is ${ }_{426}$ (i) collective, based on contact inhibition of locomotion, and ${ }_{427}$ (ii) directed, due to the presence of an external gradient. In its ${ }_{428}$ simplest form-point-like cells in one dimension-we have ${ }_{429}$ mapped the model to the lattice gas model of statistical physics, ${ }_{430}$ which affords analytic results for the migration velocity. We ${ }_{431}$ have seen that an optimal cell-cell adhesion strength emerges 432 that maximizes migration velocity, and that this optimum ${ }_{433}$ depends on the interplay between the motility forces and the ${ }_{434}$ configurational statistics of the cells. In its more realistic ${ }_{435}$ form - spatially extended cells embedded in ECM in two ${ }_{436}$ dimensions-we have seen that the optimum exists for both ${ }_{437}$ cell-cell and cell-ECM adhesion strengths. Clusters with ${ }_{438}$ intermediate adhesion are fastest because they are the most 439 fluid: they are intact, extended in the gradient direction, and ${ }_{440}$ maximally variable in cluster shape.

${ }_{441}$ Our prediction that there exist optimal cell-cell and cell${ }_{442}$ ECM adhesion strengths could be tested experimentally. Ex${ }_{443}$ periments suggest that both cell-cell and cell-ECM adhesion ${ }_{444}$ are crucial for tumor invasion, as well as for homeostasis ${ }_{445}$ in healthy tissues (35). Experimental perturbations could be ${ }_{446}$ used to modulate cadherin or integrin levels to tune cell-cell or ${ }_{447}$ cell-ECM adhesion respectively, and the effects on migration ${ }_{448}$ velocity could be investigated. For example, downregulation 449 of E-cadherin within a tumor spheroid was recently achieved ${ }_{450}$ by introduction of interstitial flow, which was subsequently ${ }_{451}$ seen to promote tumor invasion (36).

Our observation that variability in aspect ratio correlates ${ }_{453}$ with migration velocity could also serve as a phenomenologi${ }_{454} \mathrm{cal}$ signature to look for in experiments. Variability in cluster ${ }_{455}$ shape is straightforward to extract from microscopy videos ${ }_{456}$ and quantify, and it abstracts away the underlying molecular ${ }_{457}$ details of the adhesion or migration. It would be interesting ${ }_{458}$ to see whether the fastest clusters are generically the most 459 fluid across biological systems, regardless of the nature of the 460 molecular perturbation applied.

${ }_{461}$ We have considered only one- and two-dimensional migra${ }_{462}$ tion, whereas three-dimensional migration is clearly prevalent, 
${ }_{463}$ rich in its modalities (e.g., mesenchymal, amoeboid, lobopo- ${ }_{513}$ ${ }_{464} \mathrm{dial}$ ), and dependent on tunable factors (e.g., adhesion, cell ${ }_{514}$ ${ }_{465}$ confinement, contractility, deformability, proteolytic capacity) 515 466 (37-39). It would be possible in the future to extend our ${ }_{467}$ model to three dimensions to investigate some of these factors ${ }_{468}$ and migration modes. Nonetheless, important examples of ${ }^{517}$ ${ }_{469} 1 \mathrm{D}$ and 2D migration exist, to which our findings may more ${ }^{518}$ ${ }_{470}$ directly apply. Examples of 1D or quasi-1D migration include ${ }^{519}$ ${ }_{471}$ preferential migration of tumor cells, cancer stem cells, and ${ }^{520}$ 472 leukocytes along a bundle of linear collagen fibrils $(40,41)$, as ${ }_{473}$ well as migration of fibroblasts on $1 \mathrm{D}$ fibril-like lines $(37,42){ }_{522}{ }_{521}$ ${ }_{474}$ Examples of 2D or quasi-2D migration include wound healing ${ }_{523}$ 475 (or gap closure) in an epithelial tissue, cells migrating on a ${ }_{476}$ bone, migration of single epithelial cells along 2D sheets of 477 basement membranes, and patrolling of leukocytes along the ${ }_{478}$ luminal surface of blood vessels (43-46). ${ }_{479}$ Our observation that cluster fluidity maximizes migration ${ }_{527}$ ${ }_{480}$ velocity is a purely mechanical effect: intermediate adhesion ${ }_{528}$ ${ }_{481}$ promotes cluster configurations that maximize net motility ${ }_{529}$ ${ }_{482}$ forces in the gradient direction. Previous work has also shown ${ }_{530}$ ${ }_{483}$ that cluster fluidity improves gradient sensing due to a different ${ }_{484}$ mechanism: fluidity averages out detection noise due to cell-to- ${ }^{531}$ ${ }_{485}$ cell variability (34). We do not consider detection noise $\left(5,23,{ }^{532}\right.$ $\left.{ }_{486} 34\right)$ or cell-to-cell variability (34) here. It would be interesting ${ }^{533}$ ${ }_{487}$ to investigate how these distinct advantages of cluster fluidity ${ }_{534}$ ${ }_{488}$ act in concert or whether they combine synergistically.

489 The model developed here is generic, minimal, and not 490 specific to any particular cell type. In general, there can be ${ }_{491}$ more than one cell type within a single cluster. In that case, it ${ }_{537}$ ${ }_{492}$ is straightforward to extend our model to include a set of cell- ${ }_{538}$ ${ }_{493}$ cell interaction parameters $\gamma_{i j}$ between every pair of cell types ${ }_{539}$ ${ }_{494} i$ and $j$, or a set of cell-ECM interaction parameters $\alpha_{k}$ for ${ }_{495}$ each cell type. We have considered only the simplest version ${ }_{496}$ of this scenario here, but it may be interesting in the future 497 to generalize our work to systems that exhibit heterogeneous ${ }_{498}$ collective migration.

\section{${ }_{499}$ AUTHOR CONTRIBUTIONS}

${ }_{500}$ UR and AM designed and performed the research. UR con501 tributed analytic tools and analyzed data. UR and AM wrote 502 the manuscript.

\section{${ }_{503}$ ACKNOWLEDGMENTS}

${ }_{504}$ UR thanks Michael Vennettilli for valuable inputs in the ana505 lytical treatment. UR acknowledges helpful discussions with ${ }_{506}$ Raj Kumar Manna and Saikat Sinha regarding computational 507 techniques. This work was supported by Simons Foundation ${ }_{508}$ Grant No. 376198.

\section{${ }_{509}$ REFERENCES}

1. Collins C. and W J. Nelson. 2015. Running with neigh- ${ }^{557}$ bors: coordinating cell migration and cell-cell adhesion ${ }_{558}$ Curr. Op. Cell Biol. 36:62-70.
2. Friedl P. and D. Gilmour. 2009. Collective cell migration in morphogenesis, regeneration and cancer. Nat. Rev. 10:445-457.

3. Anona E., X. Serra-Picamalb, P. Hersen, N. C. Gauthier, M. P. Sheetz, X. Trepat, and B. Ladoux. 2012. Cell crawling mediates collective cell migration to close undamaged epithelial gaps. Proc. Natl. Acad. Sci. USA. 109:10891-10896.

4. Theveneau, E., L. Marchant, S. Kuriyama, M. Gull, B. Moepps, M. Parsons, and R. Mayor. 2010. Collective chemotaxis requires contact-dependent cell polarity. Dev. Cell. 19:39-53.

5. Ellison D., A. Mugler, M. D. Brennana, S. H. Leeb, R. J. Huebnere, E. R. Shamire, L. A. Wooa, J. Kima, P. Amarf, I. Nemenman, A. J. Ewalde, and A. Levchenko. 2016. Cell-cell communication enhances the capacity of cell ensembles to sense shallow gradients during morphogenesis. Proc. Natl. Acad. Sci. USA. 113:E679-E688

6. Mayor, R., and C. Carmona-Fontaine. 2010. Keeping in touch with contact inhibition of locomotion. Trends Cell Biol. 20: 319-328.

7. Pokutta S. and W. I. Weis. 2007. Structure and mechanism of cadherins and catenins in cell-cell contacts. Annu. Rev. Cell. Dev. Biol.. 23:237-261.

8. Zamir E. and B. Geiger. 2001. Molecular complexity and dynamics of cell-matrix adhesions. J. Cell Sci..114:35833590 .

9. Canel M., A. Serrels, M. C. Frame, and V. G. Brunton. 2013. E-cadherin-integrin crosstalk in cancer invasion and metastasis J. Cell Sci. 126: 393-401.

10. Richardson, J., A. Gauert, L. B. Montecinos, L. Fanlo, Z. M. Alhashem, R. Assar, E. Marti, A. Kabla, S. Härtel, and C. Linker. 2016. Leader cells define directionality of trunk, but not cranial, neural crest cell migration. Cell. Rep. 15: 2076-2088.

11. Agata Fronczak. 2013. Cluster properties of the onedimensional lattice gas: the microscopic meaning of grand potential. Phys. Rev. E 87: 022131.

12. Graner F. and J. A. Glazier. 1992. Simulation of Biological Cell Sorting Using a Two-Dimensional Extended Potts Model. Phys. Rev. Lett. 69: 2013.

13. Glazier J. A. and F. Graner. 1993. Simulation of the differential adhesion driven rearrangement of biological cells. Phys. Rev. E 47: 2128.

14. Camley B. A. and W-J Rappel. 2017. Physical models of collective cell motility: from cell to tissue. J. Phys. D: App. Phys. 50: 113002. 
15. Agata Fronczak. 2012. Microscopic meaning of grand 605 potential resulting from combinatorial approach to a 600 general system of particles. Phys. Rev. E 86: 041139. ${ }_{607}$

16. Ermentrout, G. B. and L. Edelstein-Keshet. 1993. Cellular automata approaches to biological modeling. $J .609$ Theor. Biol. 160:97-133.

17. Maire, T. and H. Youk. 2015. Molecular-level tuning of 612 cellular autonomy controls the collective behaviors of cell populations. Cell Syst. 1:349-360.

18. Mente, C., A. Voss-Böhme, and A. Deutsch. 2015. 615 Analysis of individual cell trajectories in lattice-gas 616 cellular automaton models for migrating cell populations. Bull. Math. Biol. 77:660-697.

19. Szabó, A., R., Ünnep, E. Méhes, W. O. Twal, W. S. ${ }^{619}$ Argraves, Y. Cao, and A. Czirók. 2010. Collective cell ${ }^{620}$ motion in endothelial monolayers. Phys. Biol. 7:046007. ${ }^{621}$

20. Albert P. J. and U. S. Schwarz. 2016. Dynamics of Cell Ensembles on Adhesive Micropatterns: Bridging the Gap between Single Cell Spreading and Collective Cell Migration. PLoS Comput. Biol. 12:e1004863.

21. Kabla, A. J. 2012. Collective cell migration: leader- ${ }^{626}$ ship, invasion and segregation. J. R. Soc. Interface. ${ }_{628}^{627}$ 9:3165-3183.

Maclaren, O. J., H. M. Byrne, A. G. Fletcher, and P. ${ }_{629}^{62}$ K. Maini. 2015. Models, measurement and inference in epithelial tissue dynamics. Math. Biosci. Eng. 12 (6):1321-1340.

23. Varennes, J. and B. Han, A. Mugler. 2016. Collective ${ }_{634}$ Chemotaxis through Noisy Multicellular Gradient Sens- 635 ing. Biophys. J. 111: 640-649.

24. Carmona-Fontaine C., H. K. Matthews, S. Kuriyama, ${ }^{637}$ M. Moreno, G. A. Dunn, M. Parsons, C. D. Stern, and 638 R. Mayor. 2008. Contact inhibition of locomotion in 639 vivo controls neural crest directional migration. Nature (London) 456: 957.

25. Desai R. A., S. B. Gopal, S. Chen, and C. S. Chen. 2013. Contact inhibition of locomotion probabilities drive solitary versus collective cell migration. J. R. Soc. Interface 10: 20130717.

26. Bianco A., M. Poukkula, A. Cliffe, J. Mathieu, C. M. Luque, T. A. Fulga, and P. Rørth. 2007. Two distinct modes of guidance signalling during collective migration of border cells. Nature (London) 448: 362.

27. Rørth P. 2007. Collective guidance of collective cell 650 migration. Trends Cell Biol. 17: 575.
28. Inaki M., S. Vishnu, A. Cliffe, and P. Rørth. 2012. Effective guidance of collective migration based on differences in cell states. Proc. Natl. Acad. Sci. U.S.A. 109: 2027.

29. Wang X., L. He, Y. I. Wu, K. M. Hahn, and D. J. Montell. 2010. Light-mediated activation reveals a key role for Rac in collective guidance of cell movement in vivo. Nat. Cell Biol. 12: 591.

30. Malet-Engra G., W. Yu, A. Oldani, J. Rey-Barroso, N. S. Gov, G. Scita, and L. Dupré. 2015. Collective cell motility promotes chemotactic prowess and resistance to chemorepulsion. Curr. Biol. 25: 242.

31. Camley, B. A., J. Zimmerman, H. Levine, and W.-J. Rappel. 2016. Collective signal processing in cluster chemotaxis: roles of adaptation, amplification, and coattraction in collective guidance. PLoS Comput. Biol. 12(7): e1005008

32. Camley, B. A., J. Zimmerman, H. Levine, and W.-J. Rappel. 2016. Emergent Collective Chemotaxis without Single-Cell Gradient Sensing. Phys. Rev. Lett. 116:098101.

33. Dapeng B., X. Yang, M. C. Marchetti, and M. L. Manning. 2016. Motility-Driven Glass and Jamming Transitions in Biological Tissues. Phys. Rev. X 6: 021011.

34. Camley B. A. and W.-J. Rappel. 2017. Cell-to-cell variation sets a tissue-rheology-dependent bound on collective gradient sensing. Proc. Natl. Acad. Sci. 114 (47): E10074-E10082.

35. Janiszewska M., M. C. Primi, and T. Izard. 2020. Cell adhesion in cancer: Beyond the migration of single cells. J. Biol. Chem. 295(8):2495-2505.

36. Huang Y. L., Y. Ma, C. Wu, C. Shiau, J. E. Segall, and M. M. Wu. 2020. Tumor spheroids under perfusion within a 3D microfluidic platform reveal critical roles of cell-cell adhesion in tumor invasion. Sci. Rep. 10:9648.

37. Yamada K. M. and M. Sixt. 2019. Mechanisms of 3D cell migration. Nat. Rev. 20: 738-752.

38. Liu Y. J. et al. 2015. Confinement and low adhesion induce fast amoeboid migration of slow mesenchymal cells. Cell 160: 659-672.

39. Callan-Jones A. C. and R. Voituriez. 2016. Actin flows in cell migration: from locomotion and polarity to trajectories. Curr. Opin. Cell Biol. 38, 12-17.

40. Provenzano P. P., K. W. Eliceiri, J. M. Campbell, D. R. Inman, J. G. White, and P. J. Keely. 2006. Collagen reorganization at the tumor-stromal interface facilitates local invasion. BMC Med. 4: 38. 
41. Boissonnas A., L. Fetler, I. S. Zeelenberg, S. Hugues, and S. Amigorena. 2007. In vivo imaging of cytotoxic T cell infiltration and elimination of a solid tumor. J. Exp. Med. 204, 345-356.

42. Doyle A. D., F. W. Wang, K. Matsumoto, and K. M. Yamada. 2009. One-dimensional topography underlies three-dimensional fibrillar cell migration. J. Cell Biol. 184, 481-490.

43. B. Weigelin, G. J. Bakker and P. Friedl. 2012. Intravital third harmonic generation microscopy of collective melanoma cell invasion: Principles of interface guidance and microvesicle dynamics. Intravital 1: 32-43.

44. Fisher G., and L. Rittie. 2018. Restoration of the basement membrane after wounding: a hallmark of young human skin altered with aging. J. Cell Commun. Signal 12: 401-411.

45. Hsu J. C., Hyun K., J. S. Harunaga, K. Matsumoto, A. D. Doyle, and K. M. Yamada 2013. Region-specific epithelial cell dynamics during branching morphogenesis. Dev. Dyn. 242: 1066-1077.

46. Auffray C., D. Fogg, M. Garfa, G. Elain, O. JoinLambert, S. Kayal, S. Sarnacki, A. Cumano, G. Lauvau, and F. Geissmann. 2007. Monitoring of blood vessels and tissues by a population of monocytes with patrolling behavior. Science 317: 666-670. 